Article

\title{
In Vitro Evaluation of Different Prebiotics on the Modulation of Gut Microbiota Composition and Function in Morbid Obese and Normal-Weight Subjects
}

\author{
Alicja M. Nogacka ${ }^{1,2, *}\left(\mathbb{D}\right.$, Nuria Salazar ${ }^{1,2}$, Silvia Arboleya ${ }^{1,2}{ }^{\infty}$, Patricia Ruas-Madiedo ${ }^{1,3}(\mathbb{D}$, \\ Leonardo Mancabelli ${ }^{4}$, Adolfo Suarez ${ }^{2,5}$, Ceferino Martinez-Faedo 6,7, Marco Ventura ${ }^{4}$, \\ Takumi Tochio ${ }^{8}$, Katsuaki Hirano ${ }^{8}$, Akihito Endo ${ }^{9}$, Clara G. de los Reyes-Gavilán ${ }^{1,2}$ (I) and \\ Miguel Gueimonde ${ }^{1,2}$ (1) \\ 1 Department of Microbiology and Biochemistry of Dairy Products, Instituto de Productos Lácteos de \\ Asturias (IPLA-CSIC), 33300 Villaviciosa, Asturias, Spain; nuriasg@ipla.csic.es (N.S.); \\ silvia.arboleya@ipla.csic.es (S.A.); ruas-madiedo@ipla.csic.es (P.R.-M.); \\ greyes_gavilan@ipla.csic.es (C.G.d.l.R.-G.); mgueimonde@ipla.csic.es (M.G.) \\ 2 Diet, Human Microbiota and Health Group, Institute of Health Research of the Principality of \\ Asturias (ISPA), 33011 Oviedo, Spain; adolfo.suarez@hcabuenes.es \\ 3 Functionality and Ecology of Beneficial Microorganisms, Institute of Health Research of the Principality of \\ Asturias (ISPA), 33011 Oviedo, Spain \\ 4 Laboratory of Probiogenomics, Department of Life Sciences, University of Parma, 43121 Parma, Italy; \\ leonardo.mancabelli@genprobio.com (L.M.); marco.ventura@unipr.it (M.V.) \\ 5 Digestive Service, Central University Hospital of Asturias (HUCA), 33011 Oviedo, Asturias, Spain \\ 6 Endocrinology and Nutrition Service, Central University Hospital of Asturias (HUCA), 33011 Oviedo, \\ Asturias, Spain; ceferinofaedo@gmail.com \\ 7 Endocrinology, Nutrition, Diabetes and Obesity Group, Institute of Health Research of the Principality of \\ Asturias (ISPA), 33011 Oviedo, Spain \\ $8 \quad \beta$-Food Sciences Co., Chita 478-0046, Japan; t-tochio@bfsci.co.jp (T.T.); k-hirano@bfsci.co.jp (K.H.) \\ 9 Department of Food and Cosmetic Science, Tokyo University of Agriculture, Abashiri 099-2493, Japan; \\ a3endou@nodai.ac.jp \\ * Correspondence: alicja.nogacka@ipla.csic.es
}

Received: 20 December 2019; Accepted: 28 January 2020; Published: 30 January 2020

\begin{abstract}
The gut microbiota remains relatively stable during adulthood; however, certain intrinsic and environmental factors can lead to microbiota dysbiosis. Its restoration towards a healthy condition using best-suited prebiotics requires previous development of in vitro models for evaluating their functionality. Herein, we carried out fecal cultures with microbiota from healthy normal-weight and morbid obese adults. Cultures were supplemented with different inulin-type fructans (1-kestose, Actilight, P95, Synergy1 and Inulin) and a galactooligosaccharide. Their impact on the gut microbiota was assessed by monitoring gas production and evaluating changes in the microbiota composition (qPCR and 16S rRNA gene profiling) and metabolic activity (gas chromatography). Additionally, the effect on the bifidobacterial species was assessed (ITS-sequencing). Moreover, the functionality of the microbiota before and after prebiotic-modulation was determined in an in vitro model of interaction with an intestinal cell line. In general, 1-kestose was the compound showing the largest effects. The modulation with prebiotics led to significant increases in the Bacteroides group and Faecalibacterium in obese subjects, whereas in normal-weight individuals, substantial rises in Bifidobacterium and Faecalibacterium were appreciated. Notably, the results obtained showed differences in the responses among the tested compounds but also among the studied human populations, indicating the need for developing population-specific products.
\end{abstract}


Keywords: in vitro model; microbiota; prebiotics; gas production; obesity; functionality; HT29; RTCA; SCFA; bifidobacterial-ITS

\section{Introduction}

The human intestinal microbiota represents a very complex and diverse microbial ecosystem that remains relatively stable during adult life [1]. However, several intrinsic and environmental factors can disrupt the microbiota composition, causing a microbiota "dysbiosis" [2]. Given the frequent association of dysbiosis with different disease states, the restoration of the microbiota through dietary modulation strategies could be a suitable approach. Among the different microbiota-modulation strategies, the administration of prebiotic supplements has been associated with health benefits to the gastrointestinal tract, cardiometabolism, mental health and mineral absorption, among others [3]. An international experts group has defined a prebiotic as a "substrate that is selectively utilized by host microorganisms conferring a health benefit" [3]. Most often, these substrates are complex carbohydrate moieties that, due to the presence of $\beta$-glycosidic bonds, are resistant to digestion during their passage through the gastrointestinal tract, reaching almost intact the large intestine, where they can be metabolized by the intestinal microbiota [4]. However, it is important to underline that resistance to digestion is not enough, and, by definition, prebiotics substrate must be selectively utilized by the microorganisms, with a subsequent promotion of health.

Traditionally, the selective nature of prebiotics have been specifically associated with the genera Bifidobacterium and Lactobacillus [3]. Nevertheless, during the last two decades, the development of culture-independent technologies has demonstrated that other intestinal microorganisms could be affected as well. Among these, some butyrate producers, such as members of Clostridium cluster XIVa and IV, have been found to be favored by prebiotic supplementation, and negative correlations were also found with some pathogenic bacteria [3,5]. These results indicate more global changes associated with prebiotics consumption than just the effects upon bifidobacteria and lactobacilli and underline the importance of considering the total microbiota when screening compounds for their prebiotic properties.

On the other hand, the metabolism of prebiotics leads to the generation, as main end-products, of bacterial fermentation, of short-chain fatty acids (SCFA), among which are acetate, butyrate, propionate and also branched SCFA (BSCFA: iso-valerate and iso-butyrate) and gases hydrogen, methane and carbon dioxide [6]. These compounds are well-known mediators of the microbiota-host interaction, playing an important role in host health $[7,8]$.

To date, the most widely studied prebiotics include fructooligosaccharides (FOS) of variable chain lengths, commercial preparations often containing a mixture of molecules [9], and galactooligosaccharides (GOS), which are being often used in studies focusing on infants [10]. However, the comparative studies on the impact of different prebiotics upon the microbiota in different population groups are still scarce [11]. In this context, the assessment of the impact of prebiotics in microbiota composition and metabolism using in vitro models as a tool for screening the most effective modulatory strategies prior to accomplish expensive and complex human interventions, is valuable [12]. In vitro models, such as fecal cultures, are broadly used. Moreover, complementing such models with gas production assessment can be used for determining the fermentation profile of prebiotics by the gut microbiota of different population groups [13-18]. Among these, obese subjects constitute an interesting target, since some studies have shown that the use of prebiotics is an effective modulatory strategy in obesity [19], and animal studies provide support for a potential beneficial effect on energy homeostasis and weight loss [20]. In mice, an inverse relationship has been established between the level of bifidobacteria and some features of the metabolic alterations linked to obesity (endotoxemia, fat mass and glucose intolerance) [21]. Some of these were confirmed in human studies, such as the increase of bifidobacterial levels after prebiotic treatment, with beneficial systemic consequences for 
obese individuals [21-24]. However, there is still limited evidence on the in vitro fermentation profiles of different prebiotic compounds by the microbiota of obese humans [25-28] and its comparison with that of normal-weight individuals. Moreover, the characteristics of the intestinal microbiota in the extreme form of obesity (morbid obesity; MOB) (BMI $\geq 40 \mathrm{~kg} / \mathrm{m}^{2}$ ) is still not completely known $[29,30]$. The variability in the response of the obese population to prebiotic and probiotic supplementation in weight loss interventions [31] and the lower microbial richness generally characterizing the microbiota of obese subjects [32] points to the gut microbiota as a target for investigation in this field.

Unfortunately, most often the in vitro screenings of prebiotic substrates have failed to consider the microbiota complexity and the potential differences on the basal microbiota composition among different human groups, with few studies selecting the best-suited compounds for defined population groups [33]. The availability of fast, easy and cheap methods, considering the influence of the basal microbiota, for assessing the fermentability and specificity of potential prebiotics would be of help in the selection of prebiotics for specific applications to human groups. In this context, it is well-known that the microbiota of obese subjects is different from that of normal-weight (NW) individuals [30,34,35], suggesting that the impact of different prebiotic compounds in these human groups may also differ, making advisable selecting the best-suited compounds for each of them. Therefore, in this study, we aimed at the evaluation of fermentative dynamics of different prebiotic substrates and the assessment of their impact on the composition and metabolic activity on the intestinal microbiota of lean and extreme obese individuals.

\section{Results}

\subsection{Gas Production and $p H$ Variations during Fermentation}

The check of gas production in real-time allowed us to assess the in vitro fermentative dynamics of the different prebiotics. The decreases in $\mathrm{pH}$ and the gas formed by fecal microbiotas of NW and MOB individuals after $24 \mathrm{~h}$ of incubation in the presence of different carbon sources are shown in Table 1.

Table 1. Cumulative gas produced $(\mathrm{mL})$ and decreases of $\mathrm{pH}$ values $(\Delta \mathrm{pH})$ after $24 \mathrm{~h}$ of incubation in fecal cultures with normal-weight (NW) and morbid obesity (MOB) microbiota. Kinetic parameters were determined using the modified-Gompertz equation, in which " $\mathrm{A}$ " represents the upper asymptote $(\mathrm{mL})$ and " $\mu$ " is the rate of gas production $(\mathrm{mL} / \mathrm{h})$. The values not sharing the same superscript $(\mathrm{a}, \mathrm{b}, \mathrm{c}$ or d) indicate significant differences ( $p$-value $<0.05$ ) among carbon sources for each population group (NW or MOB).

\begin{tabular}{|c|c|c|c|c|c|c|}
\hline Group & Condition & $\Delta \mathrm{pH}$ & Cumulative Gas & A & $\mu$ & $\mathbf{R}^{2}$ \\
\hline \multirow{8}{*}{ МOB } & Control & $0.10^{\mathrm{a}} \pm 0.06$ & $5.10^{\mathrm{a}} \pm 0.65$ & 5.111 & 0.39 & 0.979 \\
\hline & Glucose & $-1.22^{b} \pm 0.43$ & $18.44^{b} \pm 7.08$ & 19.306 & 1.205 & 0.999 \\
\hline & 1-kestose & $-1.34^{b} \pm 0.20$ & $21.05^{b} \pm 5.09$ & 22.299 & 1.267 & 0.997 \\
\hline & Actilight & $-1.37^{b} \pm 0.18$ & $19.80^{b} \pm 5.40$ & 20.486 & 1.163 & 0.998 \\
\hline & GOS & $-1.37^{b} \pm 0.20$ & $19.24^{\mathrm{b}} \pm 4.89$ & 20.394 & 1.293 & 0.998 \\
\hline & Inulin & $-0.86^{\mathrm{a}} \pm 0.17$ & $17.68^{b} \pm 8.54$ & 21.915 & 0.822 & 0.997 \\
\hline & P95 & $-1.25^{b} \pm 0.21$ & $19.82^{b} \pm 5.82$ & 21.688 & 1.294 & 0.997 \\
\hline & Synergy1 & $-1.18^{\mathrm{b}} \pm 0.10$ & $18.48^{b} \pm 8.32$ & 18.68 & 1.189 & 0.997 \\
\hline \multirow{8}{*}{ NW } & Control & $0.07^{a} \pm 0.10$ & $5.52^{\mathrm{a}} \pm 1.84$ & 5.421 & 0.364 & 0.979 \\
\hline & Glucose & $-1.16^{\mathrm{c}} \pm 0.31$ & $25.62^{c, d} \pm 6.38$ & 27.399 & 1.589 & 0.999 \\
\hline & 1-kestose & $-1.28^{c} \pm 0.16$ & $26.57^{\mathrm{d}} \pm 5.87$ & 27.52 & 1.861 & 0.999 \\
\hline & Actilight & $-1.25^{c} \pm 0.20$ & $19.73^{b, c} \pm 6.36$ & 20.635 & 1.384 & 0.998 \\
\hline & GOS & $-1.28^{\mathrm{c}} \pm 0.25$ & $22.20^{b, c, d} \pm 5.63$ & 22.641 & 1.761 & 0.998 \\
\hline & Inulin & $-0.77^{a, b} \pm 0.23$ & $19.10^{b} \pm 6.51$ & 21.662 & 0.962 & 0.997 \\
\hline & P95 & $-1.24^{c} \pm 0.16$ & $23.67^{b, c, d} \pm 5.76$ & 23.952 & 1.721 & 0.998 \\
\hline & Synergy1 & $-1.08^{b, c} \pm 0.13$ & $25.19^{b, c, d} \pm 6.25$ & 26.454 & 1.456 & 0.997 \\
\hline
\end{tabular}


The highest level of cumulative gas was reached with 1-kestose in both groups of individuals and the lowest with inulin (Table 1). Notably, in fecal cultures of MOB subjects, all prebiotics led to similar gas production $(p>0.05)$, whereas fecal cultures from NW adults showed higher heterogeneity, with significant differences in production among several substrates. The determination of kinetic parameters by the modified-Gompertz equation confirmed different dynamics of gas production between fecal cultures of MOB and NW subjects. For all prebiotics, production rates were lower in fecal cultures of MOB individuals (Table 1). In accordance with the results obtained from gas production, inulin was the substrate inducing the lower decline in $\mathrm{pH}$ in both population groups. Interestingly, the drops in $\mathrm{pH}$ did not totally mirror the increases in gas production, suggesting that differences among prebiotics are not only due to differences in their utilization yields but may also involve different microorganisms or catabolic pathways.

\subsection{Impact of Prebiotics on Microbiota Composition}

The microbiota composition was evaluated at the relative (16S rRNA gene profiling; Figure 1) and absolute level (quantification of representative microbial groups by qPCR; Figure 2) before (time 0 ) and after $24 \mathrm{~h}$ of incubation of fecal cultures with the carbohydrates.

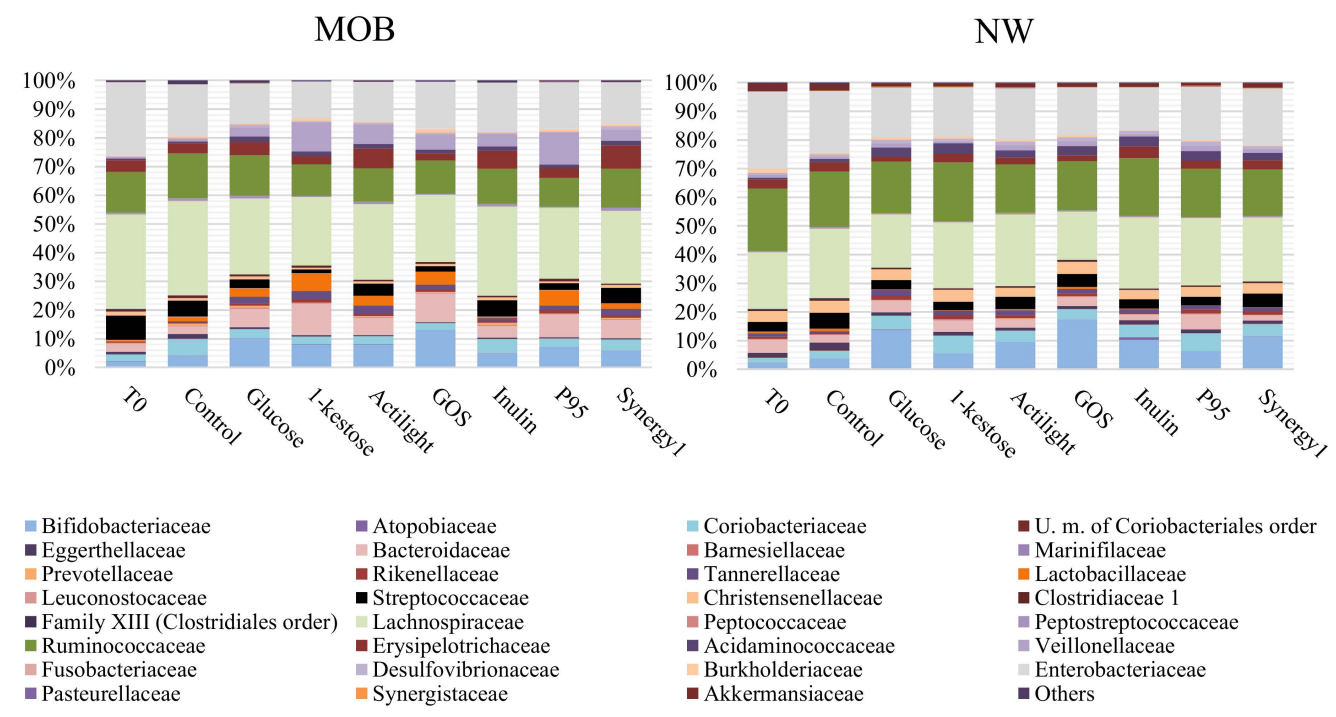

Figure 1. Microbial composition (relative abundance \%) evaluated by $16 \mathrm{~S}$ rRNA gene profiling at family levels in basal conditions (time 0 : T0) and after $24 \mathrm{~h}$ of incubation in fecal cultures with several carbon sources and without an external carbon source added (Control) in morbid obesity (MOB) and normal-weight (NW) groups.

The assessment of the microbial composition of fecal preparations before incubation (time 0 ) evidenced a high variability (Supplementary Table S1), which is an inherent feature derived from the different microbiota composition of fecal donors [36]. In spite of this, the carbon sources tested (prebiotics and glucose) displayed differential effects on the microbiota, as depending on the substrate itself and on the groups of fecal donors, MOB or NW.

Regarding the comparison among prebiotics, in fecal cultures of MOB subjects, none of the compounds affected the overall microbiota composition, without noticing any statistically significant differences among them at phyla levels (Supplementary Table S1). At family levels, significant differences with regard to the control culture were found for some minority microbial groups. These included reduced levels of the family Eggerthellaceae, belonging to Actinobacteria phylum, and an increase of the Tannerellaceae family, belonging to Bacteroidetes, in all carbon sources. Moreover, a nonsignificant trend towards higher levels of Bacteroidetes phylum and the Bacteroidaceae family were also found (Supplementary Table S1). As with regard to qPCR data, all substrates but inulin led to a significant increase of the absolute levels of the Bacteroides group, as compared to the control. This effect 
was more pronounced with 1-kestose and GOS $(8.13 \pm 0.51$ and $8.17 \pm 0.55 \mathrm{Log}$ CFU/mL, respectively) (Figure 2). An increase in the absolute levels of Faecalibacterium were also obtained with all substrates, whereas the genus Bifidobacterium was not significantly affected by any prebiotic or glucose. In spite of this, when looking at specific bifidobacterial species, the absolute levels determined by qPCR of the species Bifidobacterium longum were found to be increased after incubation with glucose, 1-kestose and $\operatorname{GOS}(6.52 \pm 0.82,6.66 \pm 1.27$ and $7.13 \pm 1.03 \mathrm{Log} C F U / \mathrm{mL}$, respectively), as compared to the control $(5.47 \pm 0.31 \mathrm{Log} \mathrm{CFU} / \mathrm{mL}$ ) (Figure 2). Moreover, ITS-sequencing allowed detecting a decrease of the initial higher relative abundances of Bifidobacterium animalis subsp. lactis and Bifidobacterium crudilactis after incubation with all prebiotics tested and with glucose (Supplementary Table S1). These results indicate that in spite of no variations found at the genus Bifidobacterium levels, some changes occurred in the species profiles after incubation with the prebiotic carbohydrates.

A) MOB

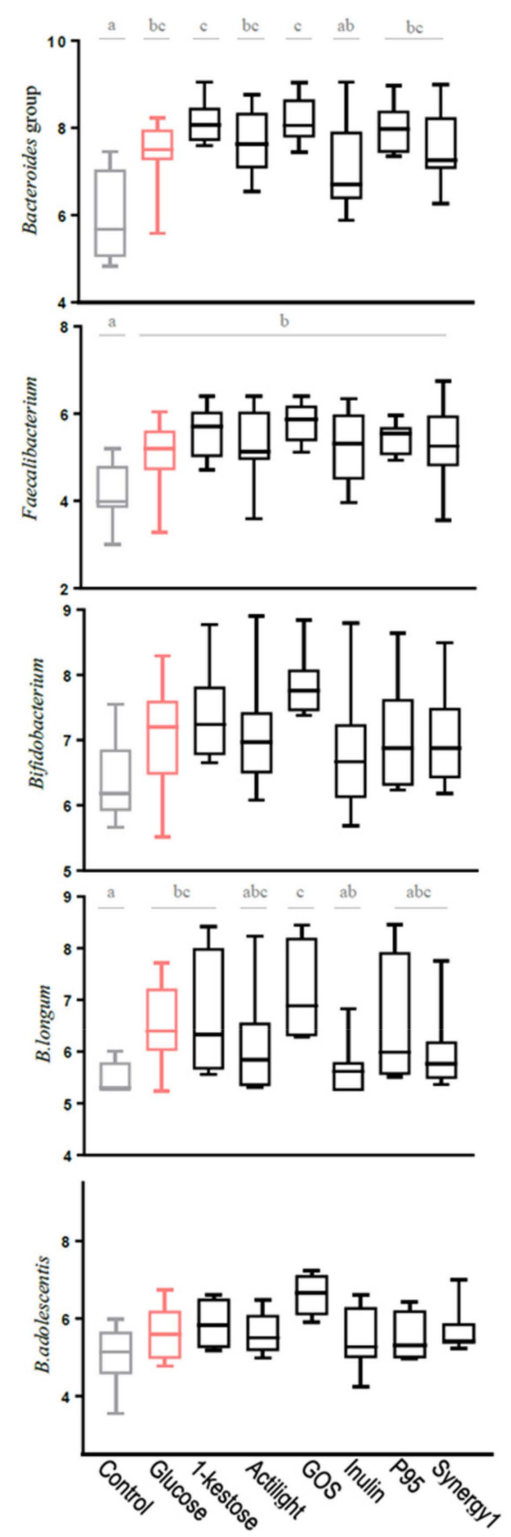

B) NW

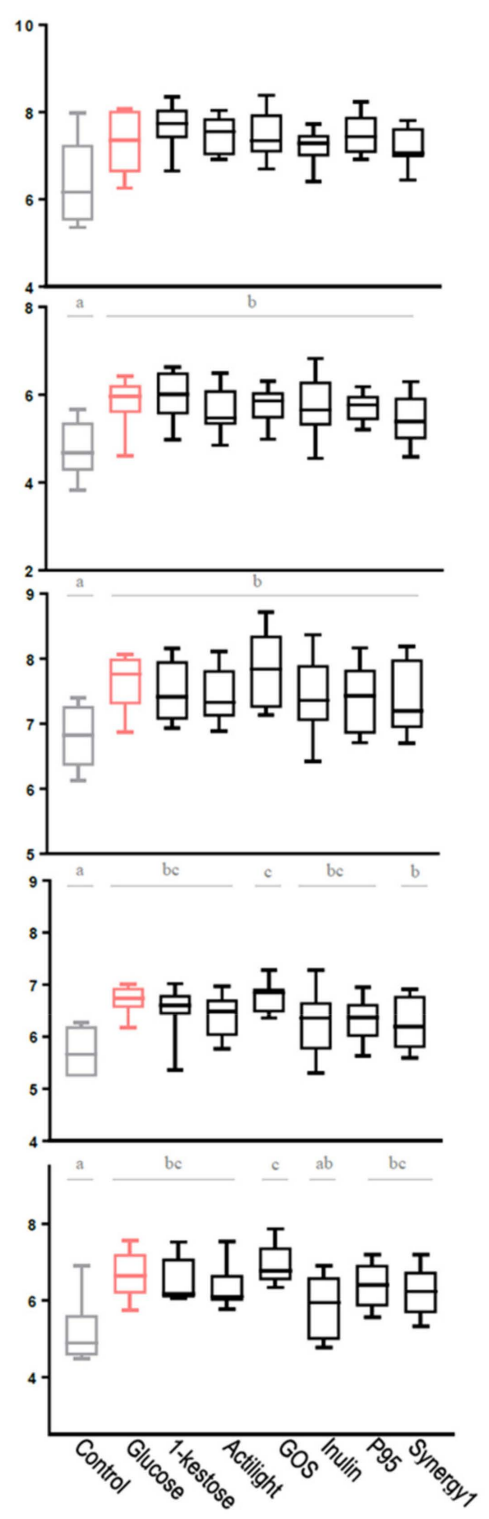

Figure 2. Absolute levels ( $\log \mathrm{CFU} / \mathrm{mL}$ ) of fecal microbial groups determined by qPCR after fecal cultures of (A) MOB and (B) NW subjects. For each microbial group, the box and whiskers plot represent median, interquartile range and minimum and maximum values obtained in each human group (NW or MOB). Different letters above the boxes indicate significant differences ( $p$-value $<0.05$ ) among carbon sources for the microbial groups considered. 
Regarding the comparison among prebiotics in fecal cultures of NW people, the 16S rRNA gene profiling evidenced very low abundances of Fusobacteria and Fusobacteriaceae in the negative control, which were practically undetectable after incubation with prebiotics (Supplementary Table S1). qPCR analyses showed that all carbon sources (prebiotics and glucose) caused a significant increase of the absolute levels of Faecalibacterium and Bifidobacterium at $24 \mathrm{~h}$ of incubation but did not significantly affect the population of Bacteroides (Figure 2). In addition, the absolute levels of the species B. longum increased significantly in fecal cultures of NW people with all substrates tested, and the same was true for Bifidobacterium adolescentis, with the exception of the prebiotic inulin (Figure 2B). Moreover, ITS-sequencing evidenced a reduction of Bifidobacterium breve relative abundances after incubation of NW fecal cultures with all substrates assayed (Supplementary Table S1).

Focusing on the comparison among fecal cultures of NW and MOB subjects, the absolute quantification (qPCR) of the main bacterial groups evidenced significant differences in the basal microbiota composition (time 0 ), for most microorganisms analyzed, between MOB and NW individuals (Supplementary Table S2). Moreover, the alpha-diversity (Chao-1 index) determined with the $16 \mathrm{~S}$ rRNA gene profiling data demonstrated a reduced diversity $(p<0.05)$ in MOB subjects, as compared to NW (132.85 \pm 43.39 vs. $169.19 \pm 21.29$, respectively). In the cultures with glucose, the differences in favor of the NW cultures for the microbial groups Akkermansia, Faecalibacterium, B. adolescentis and Clostridium cluster XIVa were maintained along incubation, giving rise to significantly higher counts of these microorganisms in NW cultures, as compared to MOB. Among prebiotics, inulin contributed to maintain differences already existing in the basal population for Bifidobacterium catenulatum in favor of the fecal cultures of NW individuals. The genus Akkermansia was significantly higher at $24 \mathrm{~h}$ of incubation in the cultures of NW individuals with respect to MOB in all conditions, these differences being not evident in cultures of the basal microbiota with no carbohydrates added (negative control). However, the most noticeable effect among prebiotics was that promoted by GOS on the Bacteroides group in MOB. Interestingly, ITS analysis showed a clear differential pattern of abundances of several bifidobacterial species between fecal cultures of NW and MOB subjects after incubation with different carbon sources (Supplementary Figure S1). The comparison between fecal samples of both human population groups in basal conditions (time 0 , before incubation) reflected higher abundances of Bifidobacterium mongoliense and B.crudilactis in NW individuals $(2.78 \pm 4.13 \%$ and $14.98 \pm 17.04 \%$, respectively) than in MOB subjects $(0.16 \pm 0.16 \%$ and $1.96 \pm 1.46 \%$, respectively) and a lower species richness (number of species of bifidobacteria detected by ITS-sequencing) in the NW fecal cultures at time 0 and after $24 \mathrm{~h}$ incubation in all conditions assayed (Supplementary Table S3). Moreover, the qPCR quantification of bifidobacterial species confirmed higher $(p<0.05)$ levels of B. longum (NW: $5.85 \pm 0.48$; MOB: $5.60 \pm 0.32$ ) and B. catenulatum (NW: $6.80 \pm 0.70$; MOB: $5.34 \pm 1.10$ ) in NW subjects. All these results point to substantial differences at the species level in the microbiota of NW and MOB subjects that are conditioning differences among fecal cultures from groups NW and MOB subjects after the incubation with prebiotics.

\subsection{Production of Short-chain Fatty Acids}

In a similar way as for microbiota composition, differences on the levels of SCFA were determined in fecal cultures depending on the prebiotic tested and the population group considered (Figure 3). Focusing on the comparison among prebiotics, GOS and 1-kestose were the substrates promoting the highest increase of total SCFA at $24 \mathrm{~h}$ of incubation in NW and MOB fecal cultures, respectively. 1-kestose gave rise to the highest increase of acetate and propionate among prebiotics tested in fecal cultures of MOB subjects, whereas GOS was the main promoter of acetate production in cultures from $\mathrm{NW}$ individuals. Inulin and Synergy1 were the prebiotics with a lower impact on the production of acetate and propionate in fecal cultures of both MOB and NW people. All prebiotics enhanced butyrate production in fecal cultures of NW individuals, with no clear differences among the different compounds (Figure 3B). In contrast, not statistically significant increases of butyrate were evidenced in fecal cultures of MOB subjects (Figure 3A), which could be due to the high variability in the production 
of this compound by the fecal cultures analyzed. In spite of that indicated above, no significant differences were obtained in the increments of acetic, propionic, butyric, BSCFA and total SCFA between fecal cultures of MOB and NW individuals at $24 \mathrm{~h}$ of incubation (Mann Whitney $\mathrm{U}$ test, $p$-value $>0.05$ ).
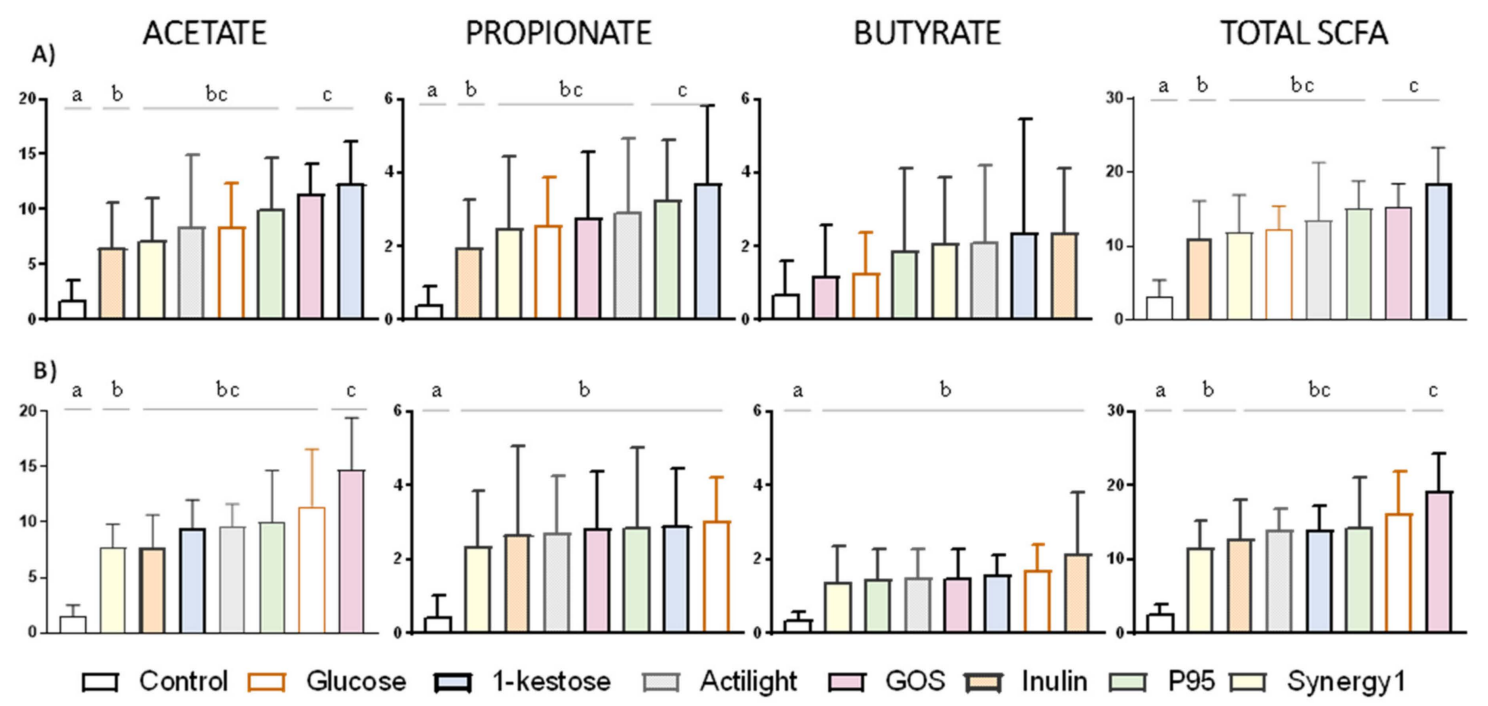

Figure 3. Increments in ascending order, with respect to time 0 , in the concentration (mM) of the major short-chain fatty acids (acetic, propionic and butyric) after $24 \mathrm{~h}$ of incubation with different carbon sources in fecal cultures from MOB (A) and NW (B) groups. Differences are shown for each short-chain fatty acid (SCFA); columns that do not share the same letter are significantly different $(p<0.05)$.

\subsection{Interaction of the Isolated Microbiotas and Supernatants from the Fecal Cultures with HT29 Cells}

Functional differences of fecal supernatants (FS) and isolated microbiotas (IM) collected before and after incubation with representative prebiotics (1-kestose, Actilight, inulin and GOS) were evaluated through an in vitro model using the HT29 intestinal cell line (Figure 4). Regarding FS, a significant decrease of the Area Under the Curve (AUC) values was evidenced after incubation of the HT29 cell line with samples from fecal MOB cultures added with prebiotics (AUC values ranging between -0.07 and 0.04$)$, with respect to the value before the addition of substrates $(0.63 \pm 0.42)$. The only exception to this was inulin, for which no significant variations were obtained (Figure $4 \mathrm{~A}$ ). It is interesting to note that AUC determined with FS from cultures of MOB subjects added with prebiotics resemble those obtained with FS of NW subjects. Notably, in these last samples, the AUC before incubation with HT29 were lower than in MOB cultures. These data suggest that the functionality of the FS from MOB subjects could be restored (becoming similar to that of NW subjects) after culturing with some of the prebiotics studied (1-kestose, Actilight and GOS). In co-cultures of HT29 with IM from fecal cultures of MOB and NW groups, all prebiotics promoted significant increases of AUC values, with the exception of inulin in both population cohorts. 

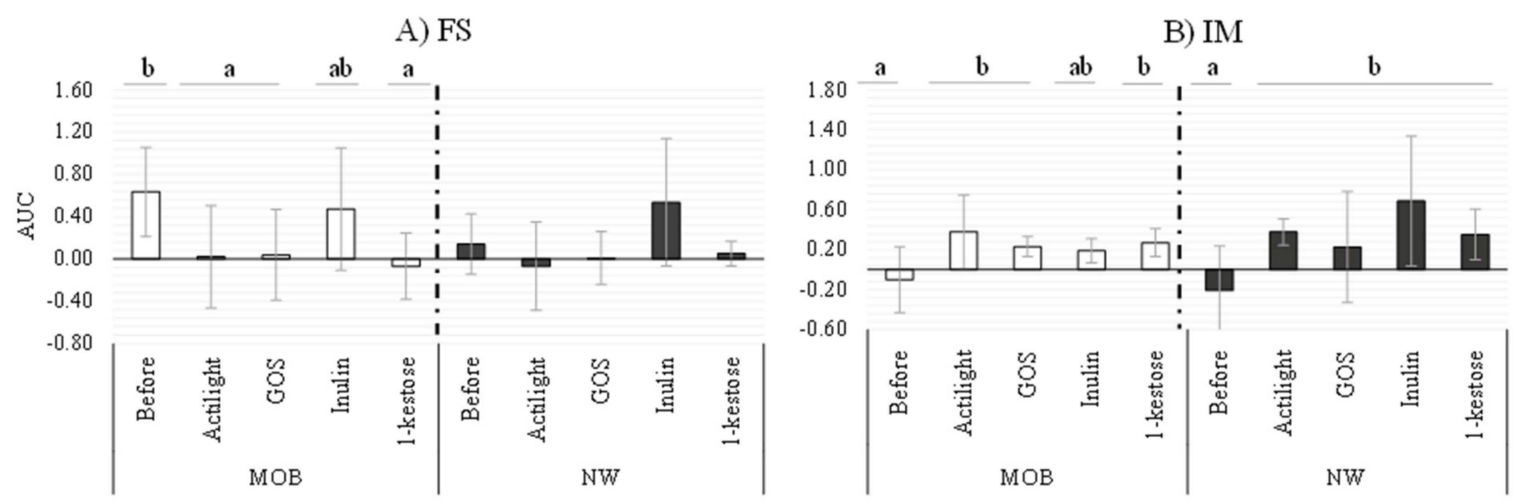

Figure 4. Real-time monitoring the interaction between (A) fecal supernatants and (B) isolated microbiota obtained before and after incubation with prebiotics and HT29 intestinal epithelial cells. Values (media $\pm \mathrm{SD}$ ) correspond to the Area Under the Curve (AUC) resulting from monitoring the cell index $(\mathrm{CI})$ during $10 \mathrm{~h}$. Significant differences $(p$-value $<0.05)$ represent the comparison of results before and after prebiotics addition in each condition.

\section{Discussion}

The definitive way to prove the impact of prebiotics on gut microbiota and health is through human intervention studies. However, these studies are expensive and time-consuming, and it is advisable to perform an initial screening of the different candidate substrates by using affordable in vitro models that could help to predict in vivo functionality [12]. In a previous work, we described an in vitro model that allowed us to predict the functionality of IM and FS of different human populations groups [37]. Its application in the present work for assessing the impact of several prebiotics in NW and MOB microbiota highlighted potential modulatory benefits on the gut microbiota of some FOS and GOS. Particularly, real-time monitoring the interaction with the HT29 intestinal cell line of FS from fecal cultures of MOB subjects added with different prebiotics evidenced a response that approaches values obtained of FS from NW cultures in the same conditions. It suggests a possible restoration of the unbalanced functionality of the microbiota by some of the prebiotic substrates tested. Nevertheless, inulin preserved a behavior more similar to the initial situation in NW and MOB groups, probably due to the fact that inulin was the prebiotic with less marked effects on microbiota composition and activity, which is consistent with previous reports by other authors $[14,16]$.

Another functional approach tested in this study was the application of a gas monitoring profiling system during fecal cultures of NW and MOB microbiotas with different prebiotics. Although this system has been recently applied to human fecal cultures [18,38], this is the first report on their use to prebiotics evaluation. Differences in gas production ability among prebiotic substrates can be partly explained by possible differences in the fermentability among compounds but also by differential effects of these substrates on the intestinal microbiota. In fact, prebiotics may differ in their ability to modulate the growth and activity of those microorganisms with limited gas production and/or to up-regulating microbial gas-consuming reactions (methanogenesis, sulfate reduction and acetogenesis) [39]. In addition to the potential direct impact on gas-producing bacteria, prebiotics are also known to affect other microorganisms of the intestinal microbiota, such as bifidobacteria, not releasing gas but producing acetate and lactate; these compounds could be involved in cross-feeding mechanisms with gas-producing microorganisms such as Clostridium spp. or sulphate-reducing bacteria [40]. Therefore, the final gas formed will depend, not only on the chemical and physical structure of the prebiotic, but on several other factors related with the composition and metabolic activity of the intestinal microbiota. In this context, different fermentation dynamics of fecal cultures from MOB and NW people were demonstrated in this study. A lower rate of gas production was appreciated with the MOB microbiota in all tested carbon sources. This feature suggests a metabolically less active microbiota. 
In the context of obesity, an inverse association between body mass index and $\mathrm{H}_{2}$ and $\mathrm{CH}_{4}$ gas detection in breath tests has been reported [41,42], which is in good agreement with the lower cumulative gas in MOB cultures obtained herein. Although emphasis has been given to the potential inflammatory or carcinogenic properties of colonic gases, emerging evidence suggests that these gases might have a beneficial effect in colonic health [43]. One of the main gases produced by anaerobic fermentation is $\mathrm{H}_{2}$ [44], and an imbalance in its metabolism $\left(\mathrm{H}_{2}\right.$-producing and $\mathrm{H}_{2}$-consuming bacteria) might facilitate inflammation [43]. It is intriguing to consider whether the promotion of gas production in MOB subjects could improve antioxidant and antiapoptotic status than contributing to decrease inflammation [45].

The prebiotics used in the present work have previously proven efficacy for modulating the microbiota of the general or specific population groups, both in in vivo and in vitro models [21]. In our case, the most pronounced effects in NW and MOB fecal cultures were obtained with 1-kestose and GOS. In this way, 1-kestose has been found to be metabolized by different intestinal microorganisms $[46,47]$. Moreover, a recent study reported that the administration to healthy volunteers of 1-kestose ( $5 \mathrm{~g} / \mathrm{day})$ during eight weeks promoted an increase of the intestinal populations of Faecalibacterium prausnitzii and Bifidobacterium spp. [48]. The potential beneficial changes promoted by GOS and FOS on the intestinal microbiota found in the present work are in good agreement with the widely reported effects of these substrates on the microbiota composition of the general population, assessed in clinical trials and used in vitro models [21,49].

It is important to emphasize that in the present work we have focused on morbid obese subjects, which could make the comparison with other studies difficult since, in the literature available, obese individuals are not often subcategorized. Moreover, the obesity-associated microbiota shifts are still not completely known, and several confounding factors often make this task difficult [32,50]. In this regard, the modulation of the microbiota of obese subjects by prebiotics has produced contradictory results on the genus Bacteroides, with increases reported after the administration of $\alpha$-glucooligosaccharide and arabinogalactan $[25,26]$ and decreases with FOS [23]. Discrepancies on experimental results are likely due to a differential effect depending on the type of prebiotics, the experimental design and the analytical techniques used to determine the composition of the intestinal microbiota by different authors. It is also necessary to point out that in the present work all prebiotics tested were able to up-modulate the levels of the genus Faecalibacterium, a microorganism with well-known anti-inflammatory properties [51] and, therefore, interesting in the context of obesity, which is generally accompanied by a low-grade inflammation $[52,53]$. A bifidogenic effect was observed only for the species B. longum after the addition of the prebiotics 1-kestose and GOS to fecal cultures. These observations and the inverse association of this species with serum lipopolysaccharides and endotoxemia [22,54] suggests that some of these prebiotics, specially 1-kestose and GOS, could be good candidates to modulate the microbiota in the context of obesity.

A broad prebiotic enhancement of absolute levels of the genus Bifidobacterium was seen in fecal cultures of the NW group, in contrast to the absence of effect in cultures from MOB subjects. In order to expand the study of the prebiotic impact on bifidobacteria, we performed an ITS-region profiling. To our knowledge, this is the first study going in detail on bifidobacteria species variation in NW and MOB fecal cultures. Even though, regarding the influence of prebiotics, the ITS-profiling only stood out as a decrease after the supplementation with carbon sources of $B$. animalis subsp. lactis and $B$. crudilactis in NW cultures and of $B$. breve in cultures of MOB subjects, a clear distinction between MOB and NW microbiotas was still evident. Firstly, we noticed a greater richness of bifidobacteria species in MOB microbiota. Additionally, differentially higher levels of B. monogoliense and B. crudilactis were present in the microbiota of the NW group, which may be explained by a higher consumption of dairy fermented foods $[55,56]$.

The production of SCFA in fecal cultures is in good agreement with variations in $\mathrm{pH}$, gas production and impact caused on the microbiota composition by the different substrates. Thus, differences in the production of propionate between fecal cultures of MOB and NW promoted by 
1-kestose could be directly related with its higher capacity (together with GOS) to differentially promote the increase of Bacteroides (the main propionate producer in the human colon and a producer of acetate) in fecal cultures of MOB individuals. Interestingly the intestinal microbiota, mainly that from $\mathrm{MOB}$ subjects, showed a better ability to produce SCFA with some of the prebiotics tested than from glucose. This could be related with the enrichment of the microbiome in some metabolic pathways involved in the initial steps of breaking down indigestible dietary polysaccharides, including pathways for starch/sucrose metabolism, galactose metabolism and butanoate metabolism, previously reported in the obese population [57].

It is also worth mentioning that the basal differences in the metabolic activity and microbiota composition, added to the specific effects of prebiotics found in the present work depending on the donor population, highlights the importance of selecting the best-suited compounds for the desired target population and the potential limitations of extrapolating conclusions from one population group to another.

To summarize, the present study provides evidence about the in vitro fermentation profiles of different prebiotics by microbiotas from NW and MOB individuals. In our study, we did not perform total metagenome analyses, but instead, we performed a microbiota characterization by 16S rRNA gene profiling and complemented it with two functional tests, gas production and interaction with an intestinal line, allowing the assessment of both microbiota compositions and some functional properties of these microbiotas. By monitoring gas production along fermentation, we found a higher capacity of gas production by fecal cultures of NW subjects than from MOB individuals. 1-kestose was the fructan showing the highest gas accumulation and largest microbiota modulation activity in MOB subjects, together with GOS, underlining the utilization of this compound by the intestinal microbiota of these individuals. The fecal cultures incubated with some of the prebiotics tested also showed differences at the functional level when assessed upon epithelial cell lines. Even though the in vitro models present inherent limitations and a difficult interpretation with respect to physiological conditions, the application of in vitro models to the analysis of microbiota composition and functionality could allow the selection of the most suitable prebiotics for different populations prior to their assessment in human intervention studies. Moreover, our results underline the interest of further exploring the prebiotic role of 1-kestose due to their modulatory capacity of the microbiota composition and activity in MOB subjects.

\section{Materials and Methods}

\subsection{Prebiotics and Carbon Sources}

Two types of prebiotics, based on their monosaccharide's composition (fructose or galactose), were evaluated. Among FOS, the trisaccharide 1-kestose ( $>99 \%$; $\beta$ Food Science Co. Ltd., Chita, Japan); Actilight ${ }^{\circledR}(\mathrm{DP}=3-5$, enzymatically produced, 95\% purity; Beghin Meiji, Lila, France); P95 (DP = 2-8, obtained by hydrolysis, 95\% purity; Beneo-Orafti, Oreye, Belgium); Synergy1 (FOS plus inulin in proportion 1:1, 92\% purity; Beneo-Orafti, Oreye, Belgium) and the long-chain fructan-inulin (DP $>36$; Sigma-Aldrich, Madrid, Spain) extracted from dahlia tubers were included in the study. A GOS from the brand Bimuno Daily (Clasado, Shinfield, England) with 79.70\% (w/w) of purity was also evaluated. Glucose (Fluka Analytical, Madrid, Spain) was also used as a nonprebiotic universal carbon source. Sterilization of all substrates was carried out by filtration through a pore size of $0.45 \mu \mathrm{m}$, except for the inulin, which was autoclaved.

\subsection{Volunteers and Fecal Sample Collection}

Fecal samples were obtained from nine healthy NW adults $\left(77.78 \%\right.$ women; BMI $\left.<25 \mathrm{~kg} / \mathrm{m}^{2}\right)$ and nine MOB volunteers ( $75 \%$ women; BMI $\geq 40 \mathrm{~kg} / \mathrm{m}^{2}$ ) recruited at the Digestive and Endocrinology Services, respectively, of Asturias Central University Hospital (HUCA, Asturias, Spain). The mean age of the volunteers was $38 \pm 9$ and $45 \pm 10$ for NW and MOB subjects, respectively. All participants have 
followed an unrestricted diet and have not taken antibiotics during the previous six months. The study was approved by the Regional Ethical Committee of Asturias Public Health Service (Ref. No 120/13, 20 November 2013), and an informed written consent was obtained from each volunteer. Samples were collected and immediately introduced into anaerobic jars (Anaerocult A System; Merck, Darmstadt, Germany) for transportation to the laboratory within $1 \mathrm{~h}$ after collection. A 1/10 (w/v) dilution was made in prereduced PBS solution and homogenized in a Lab-Blender 400 stomacher (Seward Medical, London, UK) for $5 \mathrm{~min}$.

\subsection{Fecal Batch Culture Fermentation}

Independent batch fermentations were performed at $\mathrm{pH}$-uncontrolled in a carbohydrate-free basal medium (CFBM) [58], with feces from different human donors and different carbohydrates added. Briefly, CFBM was prepared and reduced overnight in an anaerobic chamber MG500 (Don Whitley Scientific, West Yorkshire, UK) one day before the sample processing. On the day of the assay, fresh fecal samples, collected and homogenized as stated above, were added $(10 \% v / v)$ to the reduced CFBM and then were distributed into $100 \mathrm{~mL}$ bottles of the ANKOMRF system (ANKOM Technology, USA). An overnight incubation in anaerobic conditions was performed at $37^{\circ} \mathrm{C}$ prior to the addition of carbon sources in order to allow microbiota to stabilize in the culture medium.

A set of independent fermentations were performed with feces from each donor, using as carbon sources either inulin-type fructans (1-kestose, Actilight, P95, Synergy1 and inulin), GOS or glucose (nonprebiotic positive control) at a final concentration of $0.3 \%(v / v)$. A bottle with no carbon source added was used as a control. Fermentations were carried out under anaerobic conditions at $37^{\circ} \mathrm{C}$ during $24 \mathrm{~h}$. The $\mathrm{pH}$ of cultures was determined with a pHmeter (SensION + PH3, Hach; Barcelona, Spain) and was considered as an indicator of the progression of fermentation. Samples (1 mL) were taken in duplicate before incubation (time 0 ) and considered as basal conditions and at $24 \mathrm{~h}$ of incubation. Samples were centrifuged at full speed for $10 \mathrm{~min}$, and supernatants and pellets were stored separately at $-20^{\circ} \mathrm{C}$ until their use for microbiota and metabolite analyses.

\subsection{Gas Monitorization}

The cumulative gas produced along the different fermentations was monitored in real-time by using the ANKOM RF system. The system provides increases in pressure (psi), which can be converted to $\mathrm{mL}$ of gas produced using the ideal gas equation.

$$
\mathrm{V}=\mathrm{Vj} \cdot \mathrm{Ppsi} \cdot 0.068004084
$$

where $\mathrm{V}=$ gas volume at $39^{\circ} \mathrm{C}$ in $\mathrm{mL}, \mathrm{Vj}=$ headspace of digestion jar (glass bottle) in $\mathrm{mL}$ and Ppsi $=$ cumulative pressure recorded by Gas Monitor System software.

The data of gas production were fitted to a modified-Gompertz equation, a model frequently used to fit data of bacterial, plant growth, tumor proliferation and gas production [59], by using the formula:

$$
y=A \times \exp \left\{-\exp \left[\frac{\mu \times e}{A}(\lambda-t)+1\right]\right\}
$$

in which variable " $\mathrm{A}$ " represents the upper asymptote $(\mathrm{mL})$, " $\mu$ " is the rate of gas production $(\mathrm{mL} / \mathrm{h})$ and " $\lambda$ " is the time lag before the exponential phase $(h)$.

\subsection{Microbiota Composition and SCFA Quantification}

DNA was extracted from the pellets harvested using the QIAamp DNA Stool Mini kit (Qiagen $\mathrm{GmbH}$; Hilden, Germany), as previously described [60], and the isolated DNA was stored at $-20^{\circ} \mathrm{C}$ until use for qPCR analyses and $16 \mathrm{~S}$ ribosomal and intergenic ribosomal transcriber spaces (ITS). 


\subsection{1. qPCR Analyses}

Absolute levels of some relevant intestinal microbial groups (Bacteroides-Prevotella-Porphyromonas group, Lactobacillus group, Akkermansia, Clostridium cluster XIVa, Bifidobacterium and Faecalibacterium genus), as well as total bacteria, were determined at 0 and $24 \mathrm{~h}$ of fermentation by qPCR using previously described primers and conditions [31,61]. Variations in the levels of the species B. longum, B. catenulatum and B. adolescentis were assessed as described elsewhere [22].

\subsection{2. $16 \mathrm{~S}$ rRNA Gene Based Microbiota Profiling}

Purified DNA was used as a template for amplification of partial 16S rRNA gene sequences by PCR using the primers and conditions described by Milani and coworkers [62]. The obtained amplicons were then sequenced by using the MiSeq (Illumina) platform at GenProbio srl (Italy). The individual reads obtained were filtered, trimmed and processed [63]. 16S rRNA operational taxonomic units were defined at $\geq 97 \%$ sequence homology using the UCLUST tool developed by Edgar [64]. All reads were classified to the lowest possible taxonomic rank using QIIME and a reference dataset from the SILVA database [65].

\subsubsection{ITS Region-Based Profiling of Bifidobacterial Microbiota}

To gain further insight into the fecal bifidobacterial populations present in the samples and how the different prebiotics affected them, the 16S-23S internal transcriber spaces of the ribosomal DNA (ITS region) was amplified by PCR using the primer pair Probio_bif_uni/Probio_bif_rev. and further sequenced as indicated in the previous section. An improved bifidobacterial ITS database, containing all publicly available bifidobacterial genomes and a custom bioinformatics script [66], were used. Relative abundance of bifidobacterial composition filtered by a minimum presence $(\geq 1 \%)$ of each species in all databases were represented by a heatmap following instructions described elsewhere [67], centered and scaled by the "scale" function in RStudio version 1.2.5001.

\subsubsection{SCFA Analyses}

The analysis of SCFA was performed by gas chromatography (GC) in the fecal culture supernatants in order to determine the molar concentrations of three main compounds: acetate, propionate and butyrate. The remaining BSFA, namely isobutyrate and isovalerate, were also quantified and summed up for further analysis. Briefly, culture supernatants collected during the fecal fermentation $(0.250$ $\mathrm{mL}$ ) were mixed with $0.3 \mathrm{~mL}$ methanol, $0.05 \mathrm{~mL}$ internal standard solution (2-ethylbutyric $1.05 \mathrm{mg} /$ $\mathrm{mL}$ ) and $0.05 \mathrm{~mL}$ of $20 \%$ formic acid. This mixture was centrifuged, and the supernatant was used for quantification of SCFA by GC, as described previously [37]. Samples were analyzed in triplicate. Increments in molar concentrations of the main SCFA and BSCFA with respect to the time 0 were calculated for each fermentation batch with the different carbon sources.

\subsection{Monitoring the Interaction of Isolated Microbiotas (IM) and Fecal Supernatants (FS) with HT29 Cells}

Briefly, the behavior of HT29 cells monolayers in a confluent state was assessed upon exposure to IM and FS collected after incubation of fecal samples with different carbon sources by using a real-time cell analyzer (RTCA-DP) xCelligence apparatus (ACEA Bioscience Inc., San Diego, CA, USA). The culture conditions and the maintenance of the intestinal epithelial cell line HT29 (ECACC 91072201) are detailed in a previous work where the functional model was developed [37]. IM were purified from 10-fold concentrated fecal cultures by using a density gradient method previously described [68]. Purified microbiotas were inactivated by UV light exposure (15 W; Selecta, Barcelona, Spain) and adjusted to $1 \times 10^{8}$ bacteria/mL using a Neubauer-improved camera (Blau Brand, Germany).

For the functional assessment of IM and FS, HT29 monolayers in a confluent state were coincubated with $6.5 \times 10^{7}$ bacteria/mL of UV-inactivated purified microbiotas in McCoy's medium (MM) (bacteria to cell ratio 10:1). In the case of fecal supernatants, the behavior of HT29 cells monolayers was assessed 
with filtered fecal supernatants ( $\mathrm{pH}$ adjusted to $7.55 \pm 0.05$ ) and diluted $40 \%$ with MM. Additionally, a negative control consisting of MM without bacteria or fecal supernatants was included in each experiment. Each sample was tested in duplicate using two independent E-plates. The monitoring was followed for every $10 \mathrm{~min}$ under standard incubation conditions. CI values recorded were normalized by the time of the sample addition and by the control sample, as previously described [69]. For statistical comparison purposes, the "Area Under the Curve" (AUC), representing the CI values along $10 \mathrm{~h}$ of incubation for each sample, was calculated as explained in [37].

\subsection{Statistics Analyses}

Unless otherwise specified, all experimental data are reported as mean \pm standard deviation. Statistical analysis of results was performed using the software SPSS v.25 (SPSS Inc., Chicago, USA). Data were compared for the effect caused on the parameters analyzed by the addition of different carbon sources in fecal cultures from each population cohort (NW and MOB) at the end of fermentation (24 h). For variables with a normal distribution (Shapiro-Wilk test) and homoscedasticity (Levene test), one-way ANOVA followed by post hoc LSD comparison were conducted (Supplementary Table S4). In the remaining cases (variables showing non-normal distribution), a Kruskal-Wallis test followed by a post hoc Dunn's test of pairwise comparisons were applied when necessary (Supplementary Table S4). A significant $p$-value of 0.05 was used for the interpretation of results. For two-group comparisons between MOB and NW (at time 0 and after incubation with all conditions), a two-tailed Student's $t$-test or Mann-Whitney $U$ test was conducted for the evaluation of data by parametric or nonparametric contrast, respectively.

Supplementary Materials: Supplementary materials can be found at http://www.mdpi.com/1422-0067/21/3/906/s1.

Author Contributions: Conceptualization, A.E., T.T., K.H., C.G.R.-G. and M.G.; volunteers recruitment, A.S. and C.M.-F.; methodology, A.N., N.S., S.A., P.R-M., L.M., A.S. and C.M.-F.; formal analysis, A.N., N.S., S.A., L.M., M.V., C.G.R.-G. and M.G.; writing - original draft preparation, A.N., C.G.R.-G. and M.G.; writing-review and editing, A.N., N.S., S.A., P.R.-M., L.M., A.S., C.M.-F., M.V., T.T., K.H., A.E., C.G.R.-G. and M.G. All authors have read and agreed to the published version of the manuscript.

Funding: The present work was financed by the project AGL2013-43770-R (receiving funds from the Ministry of Economy and Competitiveness-MINECO and the European Union FEDER funds) and by a contract with the company $\beta$-Food Sciences (Chita, Japan). Alicja M Nogacka was the recipient of a predoctoral contract granted by MINECO (reference BES-2014-068796), and S.A. received a postdoctoral Juan de la Cierva contract (Ministry of Science, Innovation and Universities, Spain, Ref. IJCI-2017-32156). Nuria Salazar is the recipient of a postdoctoral contract awarded by the Biosanitary Research Foundation in Asturias (FINBA, Spain).

Acknowledgments: We wish to thank the volunteers participating in the study and the Scientific-Technical Service of IPLA-CSIC for carrying out the SCFA determination.

Conflicts of Interest: Takumi Tochio and Katsuaki Hirano are employees at the company $\beta$ - Food Sciences.

\section{Abbreviations}

$\begin{array}{ll}\text { AUC } & \text { Area Under the Curve } \\ \text { CI } & \text { Cell Index } \\ \text { FS } & \text { Fecal Supernatant } \\ \text { GC } & \text { Gas Chromatography } \\ \text { IM } & \text { Isolated Microbiota } \\ \text { ITS } & \text { 16S-23S internal transcriber spaces } \\ \text { LSD } & \text { Least Significant Difference post-hoc } \\ \text { MM } & \text { McCoy's medium } \\ \text { MOB } & \text { Morbid Obesity } \\ \text { NW } & \text { Normal-Weight Adults } \\ \text { qPCR } & \text { Real-time polymerase chain reaction } \\ \text { rRNA } & \text { Ribosomal ribonucleic acid } \\ \text { RTCA } & \text { Real-time Cell Analyzer }\end{array}$




\section{References}

1. Lloyd-Price, J.; Abu-Ali, G.; Huttenhower, C. The healthy human microbiome. Genome Med. 2016, 8, 51. [CrossRef]

2. Levy, M.; Kolodziejczyk, A.A.; Thaiss, C.A.; Elinav, E. Dysbiosis and the immune system. Nat. Rev. Immunol. 2017, 17, 219-232. [CrossRef] [PubMed]

3. Gibson, G.R.; Hutkins, R.; Sanders, M.E.; Prescott, S.L.; Reimer, R.A.; Salminen, S.J.; Scott, K.; Stanton, C.; Swanson, K.S.; Cani, P.D.; et al. Expert consensus document: The International Scientific Association for Probiotics and Prebiotics (ISAPP) consensus statement on the definition and scope of prebiotics. Nat. Rev. Gastroenterol. Hepatol. 2017, 14, 491-502. [CrossRef] [PubMed]

4. Vernazza, C.L.; Rabiu, A.R.; Gibson, G.R. Human Colonic Microbiology and the Role of Dietary Intervention: Introduction to Prebiotics. In Prebiotics: Development \& Application; Gibson, G.R., Rastall, R.A., Eds.; John Wiley \& Sons, Ltd.: Chichester, UK, 2006; ISBN 13-978-0-470-02313-6.

5. DeVuyst, L.; Leroy, F. Cross-feeding between bifidobacteria and butyrate-producing colon bacteria explains bifdobacterial competitiveness, butyrate production, and gas production. Int. J. Food Microbiol. 2011, 149, 73-80. [CrossRef] [PubMed]

6. Cummings, J.H.; Macfarlane, G.T. Role of intestinal bacteria in nutrient metabolism. Clin. Nutr. 1997, 16, 3-11. [CrossRef]

7. Ríos-Covián, D.; Ruas-Madiedo, P.; Margolles, A.; Gueimonde, M.; de los Reyes-Gavilán, C.G.; Salazar, N. Intestinal Short Chain Fatty Acids and their Link with Diet and Human Health. Front. Microbiol. 2016, 7, 185. [CrossRef]

8. Rowland, I.; Gibson, G.; Heinken, A.; Scott, K.; Swann, J.; Thiele, I.; Tuohy, K. Gut microbiota functions: Metabolism of nutrients and other food components. Eur. J. Nutr. 2018, 57, 1-24. [CrossRef]

9. Dominguez, A.L.; Rodrigues, L.R.; Lima, N.M.; Teixeira, J.A. An Overview of the Recent Developments on Fructooligosaccharide Production and Applications. Food Bioprocess Technol. 2014, 7, 324-337. [CrossRef]

10. Wilson, B.; Whelan, K. Prebiotic inulin-type fructans and galacto-oligosaccharides: Definition, specificity, function, and application in gastrointestinal disorders. J. Gastroenterol. Hepatol. 2017, 32, 64-68. [CrossRef]

11. Sanders, M.E.; Merenstein, D.J.; Reid, G.; Gibson, G.R.; Rastall, R.A. Probiotics and prebiotics in intestinal health and disease: From biology to the clinic. Nat. Rev. Gastroenterol. Hepatol. 2019, 16, 605-616. [CrossRef]

12. Williams, C.F.; Walton, G.E.; Jiang, L.; Plummer, S.; Garaiova, I.; Gibson, G.R. Comparative Analysis of Intestinal Tract Models. Annu. Rev. Food Sci. Technol. 2015, 6, 329-350. [CrossRef] [PubMed]

13. Ghoddusi, H.B.; Grandison, M.A.; Grandison, A.S.; Tuohy, K.M. In vitro study on gas generation and prebiotic effects of some carbohydrates and their mixtures. Anaerobe 2007, 13, 193-199. [CrossRef] [PubMed]

14. Beards, E.; Tuohy, K.; Gibson, G. Bacterial, SCFA and gas profiles of a range of food ingredients following in vitro fermentation by human colonic microbiota. Anaerobe 2010, 16, 420-425. [CrossRef] [PubMed]

15. Koecher, K.J.; Noack, J.A.; Timm, D.A.; Klosterbuer, A.S.; Thomas, W.; Slavin, J.L. Estimation and Interpretation of Fermentation in the Gut: Coupling Results from a $24 \mathrm{~h}$ Batch in Vitro System with Fecal Measurements from a Human Intervention Feeding Study Using Fructo-oligosaccharides, Inulin, Gum Acacia, and Pea Fiber. J. Agric. Food Chem. 2014, 62, 1332-1337. [CrossRef] [PubMed]

16. Hernot, D.C.; Boileau, T.W.; Bauer, L.L.; Middelbos, I.S.; Murphy, M.R.; Swanson, K.S.; Fahey, G.C. In Vitro Fermentation Profiles, Gas Production Rates, and Microbiota Modulation as Affected by Certain Fructans, Galactooligosaccharides, and Polydextrose. J. Agric. Food Chem. 2009, 57, 1354-1361. [CrossRef] [PubMed]

17. VandenAbbeele, P.; Venema, K.; Van de Wiele, T.; Verstraete, W.; Possemiers, S. Different Human Gut Models Reveal the Distinct Fermentation Patterns of Arabinoxylan versus Inulin. J. Agric. Food Chem. 2013, 61, 9819-9827. [CrossRef]

18. Yao, C.K.; Rotbart, A.; Ou, J.Z.; Kalantar-Zadeh, K.; Muir, J.G.; Gibson, P.R. Modulation of colonic hydrogen sulfide production by diet and mesalazine utilizing a novel gas-profiling technology. Gut Microbes 2018, 9, 1-13. [CrossRef]

19. Dahiya, D.K.; Puniya, M.; Shandilya, U.K.; Dhewa, T.; Kumar, N.; Kumar, S.; Puniya, A.K.; Shukla, P. Gut Microbiota Modulation and Its Relationship with Obesity Using Prebiotic Fibers and Probiotics: A Review. Front. Microbiol. 2017, 8, 563. [CrossRef]

20. Delgado, G.T.C.; Tamashiro, W.M.D.S.C. Role of prebiotics in regulation of microbiota and prevention of obesity. Food Res. Int. 2018, 113, 183-188. [CrossRef] 
21. Roberfroid, M.; Gibson, G.R.; Hoyles, L.; McCartney, A.L.; Rastall, R.; Rowland, I.; Wolvers, D.; Watzl, B.; Szajewska, H.; Stahl, B.; et al. Prebiotic effects: Metabolic and health benefits. Br. J. Nutr. 2010, 104, S1-S63. [CrossRef]

22. Salazar, N.; Dewulf, E.M.; Neyrinck, A.M.; Bindels, L.B.; Cani, P.D.; Mahillon, J.; de Vos, W.M.; Thissen, J.-P.; Gueimonde, M.; de los Reyes-Gavilán, C.G.; et al. Inulin-type fructans modulate intestinal Bifidobacterium species populations and decrease fecal short-chain fatty acids in obese women. Clin. Nutr. 2015, 34, 501-507. [CrossRef] [PubMed]

23. Dewulf, E.M.; Cani, P.D.; Claus, S.P.; Fuentes, S.; Puylaert, P.G.; Neyrinck, A.M.; Bindels, L.B.; de Vos, W.M.; Gibson, G.R.; Thissen, J.-P.; et al. Insight into the prebiotic concept: Lessons from an exploratory, double blind intervention study with inulin-type fructans in obese women. Gut 2013, 62, 1112-1121. [CrossRef]

24. Parnell, J.A.; Klancic, T.; Reimer, R.A. Oligofructose decreases serum lipopolysaccharide and plasminogen activator inhibitor-1 in adults with overweight/obesity. Obesity 2017, 25, 510-513. [CrossRef] [PubMed]

25. Aguirre, M.; Bussolo de Souza, C.; Venema, K. The Gut Microbiota from Lean and Obese Subjects Contribute Differently to the Fermentation of Arabinogalactan and Inulin. PLoS ONE 2016, 11, e0159236. [CrossRef]

26. Sarbini, S.R.; Kolida, S.; Gibson, G.R.; Rastall, R.A. In vitro fermentation of commercial $\alpha$-gluco-oligosaccharide by faecal microbiota from lean and obese human subjects. Br. J. Nutr. 2013, 109, 1980-1989. [CrossRef]

27. Sarbini, S.R.; Kolida, S.; Deaville, E.R.; Gibson, G.R.; Rastall, R.A. Potential of novel dextran oligosaccharides as prebiotics for obesity management through in vitro experimentation. Br. J. Nutr. 2014, 112, 1303-1314. [CrossRef]

28. Aguirre, M.; Jonkers, D.M.A.E.; Troost, F.J.; Roeselers, G.; Venema, K. In Vitro Characterization of the Impact of Different Substrates on Metabolite Production, Energy Extraction and Composition of Gut Microbiota from Lean and Obese Subjects. PLoS ONE 2014, 9, e113864. [CrossRef]

29. Cani, P.D. Severe obesity and gut microbiota: does bariatric surgery really reset the system? Gut 2019, 68, 5-6. [CrossRef]

30. Castaner, O.; Goday, A.; Park, Y.-M.; Lee, S.-H.; Magkos, F.; Shiow, S.-A.T.E.; Schröder, H. The Gut Microbiome Profile in Obesity: A Systematic Review. Int. J. Endocrinol. 2018, 2018, 4095789. [CrossRef]

31. Dao, M.C.; Clément, K. Gut microbiota and obesity: Concepts relevant to clinical care. Eur. J. Intern. Med. 2018, 48, 18-24. [CrossRef]

32. Aron-Wisnewsky, J.; Prifti, E.; Belda, E.; Ichou, F.; Kayser, B.D.; Dao, M.C.; Verger, E.O.; Hedjazi, L.; Bouillot, J.-L.; Chevallier, J.-M.; et al. Major microbiota dysbiosis in severe obesity: Fate after bariatric surgery. Gut 2019, 68, 70-82. [CrossRef] [PubMed]

33. Valdés, L.; Salazar, N.; González, S.; Arboleya, S.; Ríos-Covián, D.; Genovés, S.; Ramón, D.; de los Reyes-Gavilán, C.G.; Ruas-Madiedo, P.; Gueimonde, M. Selection of potential probiotic bifidobacteria and prebiotics for elderly by using in vitro faecal batch cultures. Eur. Food Res. Technol. 2017, 243, 157-165. [CrossRef]

34. Ley, R.E.; Backhed, F.; Turnbaugh, P.; Lozupone, C.A.; Knight, R.D.; Gordon, J.I. Obesity alters gut microbial ecology. Proc. Natl. Acad. Sci. USA 2005, 102, 11070-11075. [CrossRef] [PubMed]

35. Gomes, A.C.; Hoffmann, C.; Mota, J.F. The human gut microbiota: Metabolism and perspective in obesity. Gut Microbes 2018, 9, 308-325. [CrossRef] [PubMed]

36. McDonald, D.; Hyde, E.; Debelius, J.W.; Morton, J.T.; Gonzalez, A.; Ackermann, G.; Aksenov, A.A.; Behsaz, B.; Brennan, C.; Chen, Y.; et al. American Gut: An Open Platform for Citizen Science Microbiome Research. mSystems 2018, 3, e00031-18. [CrossRef]

37. Nogacka, A.M.; Ruas-Madiedo, P.; Gómez, E.; Solís, G.; Fernández, N.; Suárez, M.; Suárez, A.; Salazar, N.; de los Reyes-Gavilán, C.G.; Gueimonde, M. Real-time monitoring of HT29 epithelial cells as an in vitro model for assessing functional differences among intestinal microbiotas from different human population groups. J. Microbiol. Methods 2018, 152, 210-216. [CrossRef]

38. Rotbart, A.; Moate, P.J.; Yao, C.K.; Ou, J.Z.; Kalantar-Zadeh, K. A novel mathematical model for the dynamic assessment of gas composition and production in closed or vented fermentation systems. Sensors Actuators $B$ Chem. 2018, 254, 354-362. [CrossRef]

39. Mego, M.; Manichanh, C.; Accarino, A.; Campos, D.; Pozuelo, M.; Varela, E.; Vulevic, J.; Tzortzis, G.; Gibson, G.; Guarner, F.; et al. Metabolic adaptation of colonic microbiota to galactooligosaccharides: A proof-of-concept-study. Aliment. Pharmacol. Ther. 2017, 45, 670-680. [CrossRef] 
40. Sarbini, S.R.; Rastall, R.A. Prebiotics: Metabolism, Structure, and Function. Funct. Food Rev. 2011, 3, 93-106. [CrossRef]

41. Jung, S.E.; Joo, N.S.; Han, K.S.; Kim, K.N. Obesity is inversely related to hydrogen-producing small intestinal bacterial overgrowth in non-constipation irritable bowel syndrome. J. Korean Med. Sci. 2017, 32, 948-953. [CrossRef]

42. Wilder-Smith, C.H.; Olesen, S.S.; Materna, A.; Drewes, A.M. Breath methane concentrations and markers of obesity in patients with functional gastrointestinal disorders. United Eur. Gastroenterol. J. 2018, 6, 595-603. [CrossRef] [PubMed]

43. Carbonero, F.; Benefiel, A.C.; Gaskins, H.R. Contributions of the microbial hydrogen economy to colonic homeostasis. Nat. Rev. Gastroenterol. Hepatol. 2012, 9, 504-518. [CrossRef] [PubMed]

44. Macfarlane, G.T.; Gibson, G.R. Carbohydrate Fermentation, Energy Transduction and Gas Metabolism in the Human Large Intestine. In Gastrointestinal Microbiology; Mackie, R.I., White, B.A., Eds.; Chapman \& Hall: New York, NY, USA, 1997; pp. 269-318. [CrossRef]

45. Monteiro-Sepulveda, M.; Touch, S.; Mendes-Sá, C.; André, S.; Poitou, C.; Allatif, O.; Cotillard, A.; Fohrer-Ting, H.; Hubert, E.-L.; Remark, R.; et al. Jejunal T Cell Inflammation in Human Obesity Correlates with Decreased Enterocyte Insulin Signaling. Cell Metab. 2015, 22, 113-124. [CrossRef] [PubMed]

46. Endo, A.; Nakamura, S.; Konishi, K.; Nakagawa, J.; Tochio, T. Variations in prebiotic oligosaccharide fermentation by intestinal lactic acid bacteria. Int. J. Food Sci. Nutr. 2016, 67, 125-132. [CrossRef]

47. Ose, R.; Hirano, K.; Maeno, S.; Nakagawa, J.; Salminen, S.; Tochio, T.; Endo, A. The ability of human intestinal anaerobes to metabolize different oligosaccharides: Novel means for microbiota modulation? Anaerobe 2018, 51, 110-119. [CrossRef]

48. Tochio, T.; Kadota, Y.; Tanaka, T.; Koga, Y. 1-Kestose, the Smallest Fructooligosaccharide Component, Which Efficiently Stimulates Faecalibacterium prausnitzii as Well as Bifidobacteria in Humans. Foods 2018, 7, 140. [CrossRef]

49. Rajendran, C.K.S.R.; Okolie, C.L.; Udenigwe, C.C.; Mason, B. Structural features underlying prebiotic activity of conventional and potential prebiotic oligosaccharides in food and health. J. Food Biochem. 2017, 41, e12389. [CrossRef]

50. Duvallet, C.; Gibbons, S.M.; Gurry, T.; Irizarry, R.A.; Alm, E.J. Meta-analysis of gut microbiome studies identifies disease-specific and shared responses. Nat. Commun. 2017, 8, 1784. [CrossRef]

51. Hiippala, K.; Jouhten, H.; Ronkainen, A.; Hartikainen, A.; Kainulainen, V.; Jalanka, J.; Satokari, R. The Potential of Gut Commensals in Reinforcing Intestinal Barrier Function and Alleviating Inflammation. Nutrients 2018, 10, 988. [CrossRef]

52. Sokol, H.; Pigneur, B.; Watterlot, L.; Lakhdari, O.; Bermúdez-Humarán, L.G.; Gratadoux, J.J.; Blugeon, S.; Bridonneau, C.; Furet, J.P.; Corthier, G.; et al. Faecalibacterium prausnitzii is an anti-inflammatory commensal bacterium identified by gut microbiota analysis of Crohn disease patients. Proc. Natl. Acad. Sci. USA 2008, 105, 16731-16736. [CrossRef]

53. Machiels, K.; Joossens, M.; Sabino, J.; De Preter, V.; Arijs, I.; Eeckhaut, V.; Ballet, V.; Claes, K.; Van Immerseel, F.; Verbeke, K.; et al. A decrease of the butyrate-producing species Roseburia hominis and Faecalibacterium prausnitzii defines dysbiosis in patients with ulcerative colitis. Gut 2014, 63, 1275-1283. [CrossRef] [PubMed]

54. Cani, P.D.; Neyrinck, A.M.; Fava, F.; Knauf, C.; Burcelin, R.G.; Tuohy, K.M.; Gibson, G.R.; Delzenne, N.M. Selective increases of bifidobacteria in gut microflora improve high-fat-diet-induced diabetes in mice through a mechanism associated with endotoxaemia. Diabetologia 2007, 50, 2374-2383. [CrossRef] [PubMed]

55. Delcenserie, V.; Taminiau, B.; Gavini, F.; de Schaetzen, M.-A.; Cleenwerck, I.; Theves, M.; Mahieu, M.; Daube, G. Detection and characterization of Bifidobacterium crudilactis and B. mongoliense able to grow during the manufacturing process of French raw milk cheeses. BMC Microbiol. 2013, 13, 239. [CrossRef] [PubMed]

56. Milani, C.; Duranti, S.; Napoli, S.; Alessandri, G.; Mancabelli, L.; Anzalone, R.; Longhi, G.; Viappiani, A.; Mangifesta, M.; Lugli, G.A.; et al. Colonization of the human gut by bovine bacteria present in Parmesan cheese. Nat. Commun. 2019, 10, 1286. [CrossRef]

57. Turnbaugh, P.J.; Ley, R.E.; Mahowald, M.A.; Magrini, V.; Mardis, E.R.; Gordon, J.I. An obesity-associated gut microbiome with increased capacity for energy harvest. Nature 2006, 444, 1027-1031. [CrossRef] [PubMed]

58. Al-Tamimi, M.A.H.M.; Palframan, R.J.; Cooper, J.M.; Gibson, G.R.; Rastall, R.A. In vitro fermentation of sugar beet arabinan and arabino-oligosaccharides by the human gut microflora. J. Appl. Microbiol. 2006, 100, 407-414. [CrossRef] [PubMed] 
59. Ware, A.; Power, N. Modelling methane production kinetics of complex poultry slaughterhouse wastes using sigmoidal growth functions. Renew. Energy 2017, 104, 50-59. [CrossRef]

60. Arboleya, S.; Binetti, A.; Salazar, N.; Fernández, N.; Solís, G.; Hernández-Barranco, A.; Margolles, A.; de los Reyes-Gavilán, C.G.; Gueimonde, M. Establishment and development of intestinal microbiota in preterm neonates. FEMS Microbiol. Ecol. 2012, 79, 763-772. [CrossRef]

61. Bartosch, S.; Fite, A.; Macfarlane, G.T.; McMurdo, M.E.T. Characterization of Bacterial Communities in Feces from Healthy Elderly Volunteers and Hospitalized Elderly Patients by Using Real-Time PCR and Effects of Antibiotic Treatment on the Fecal Microbiota. Appl. Environ. Microbiol. 2004, 70, 3575-3581. [CrossRef]

62. Milani, C.; Hevia, A.; Foroni, E.; Duranti, S.; Turroni, F.; Gueimonde, M.; van Sinderen, D.; Lugli, A.; Sanchez, B.; Martı, R.; et al. Assessing the fecal microbiota: an optimized ion torrent 16S rRNA gene-based analysis protocol. PLoS ONE 2013, 8, e68739. [CrossRef]

63. Nogacka, A.; Salazar, N.; Suárez, M.; Milani, C.; Arboleya, S.; Solís, G.; Fernández, N.; Alaez, L.; Hernández-Barranco, A.M.; de los Reyes-Gavilán, C.G.; et al. Impact of intrapartum antimicrobial prophylaxis upon the intestinal microbiota and the prevalence of antibiotic resistance genes in vaginally delivered full-term neonates. Microbiome 2017, 5, 93. [CrossRef] [PubMed]

64. Edgar, R.C. Search and clustering orders of magnitude faster than BLAST. Bioinforma. Appl. Note 2010, 26, 2460-2461. [CrossRef] [PubMed]

65. Quast, C.; Pruesse, E.; Yilmaz, P.; Gerken, J.; Schweer, T.; Glo, F.O.; Yarza, P. The SILVA ribosomal RNA gene database project: Improved data processing and web-based tools. Nucleic Acids Res. 2013, 41, 590-596. [CrossRef] [PubMed]

66. Milani, C.; Lugli, G.A.; Turroni, F.; Mancabelli, L.; Duranti, S.; Viappiani, A.; Mangifesta, M.; Segata, N.; van Sinderen, D.; Ventura, M. Evaluation of bifidobacterial community composition in the human gut by means of a targeted amplicon sequencing (ITS) protocol. FEMS Microbiol. Ecol. 2014, 90, 493-503. [CrossRef] [PubMed]

67. Metsalu, T.; Vilo, J. ClustVis: A web tool for visualizing clustering of multivariate data using Principal Component Analysis and heatmap. Nucleic Acids Res. 2015, 43, W566-W570. [CrossRef]

68. Hevia, A.; Delgado, S.; Margolles, A.; Sánchez, B. Application of density gradient for the isolation of the fecal microbial stool component and the potential use thereof. Sci. Rep. 2015, 5, 16807. [CrossRef]

69. Valdés, L.; Gueimonde, M.; Ruas-Madiedo, P. Monitoring in real time the cytotoxic effect of Clostridium difficile upon the intestinal epithelial cell line HT29. J. Microbiol. Methods 2015, 119, 66-73. [CrossRef] 